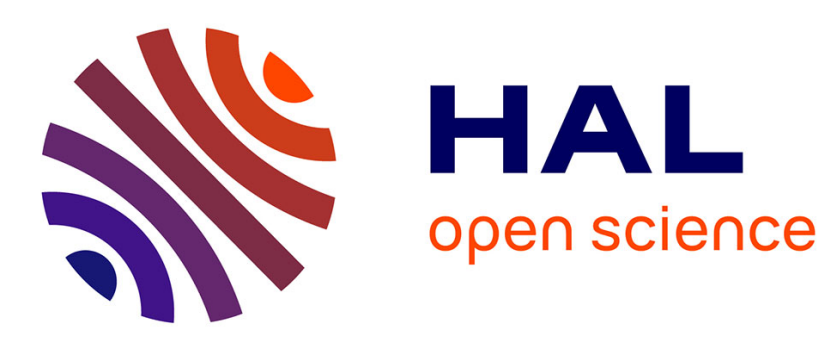

\title{
Strains and Rotations in Thin Deposited Films
}

John Price Hirth, Robert Charles Pond

\section{To cite this version:}

John Price Hirth, Robert Charles Pond. Strains and Rotations in Thin Deposited Films. Philosophical Magazine, 2010, 90 (23), pp.3129-3147. 10.1080/14786435.2010.481269 . hal-00602599

\section{HAL Id: hal-00602599 \\ https://hal.science/hal-00602599}

Submitted on 23 Jun 2011

HAL is a multi-disciplinary open access archive for the deposit and dissemination of scientific research documents, whether they are published or not. The documents may come from teaching and research institutions in France or abroad, or from public or private research centers.
L'archive ouverte pluridisciplinaire HAL, est destinée au dépôt et à la diffusion de documents scientifiques de niveau recherche, publiés ou non, émanant des établissements d'enseignement et de recherche français ou étrangers, des laboratoires publics ou privés. 


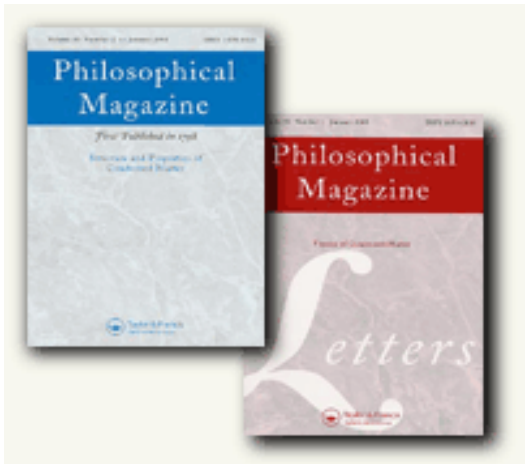

\section{Strains and Rotations in Thin Deposited Films}

\begin{tabular}{|r|l|}
\hline Journal: & Philosophical Magazine \& Philosophical Magazine Letters \\
\hline Manuscript ID: & TPHM-09-Dec-0496.R1 \\
\hline Journal Selection: & Philosophical Magazine \\
\hline Date Submitted by the & $12-$ Mar-2010 \\
\hline Complete List of Authors: & $\begin{array}{l}\text { Hirth, John; Private Individual } \\
\text { Pond, Robert; The University of Exeter, School of Engineering, } \\
\text { Mathematics and Physical Sciences }\end{array}$ \\
\hline Keywords: & $\begin{array}{l}\text { dislocation theory, epitaxy, thin films, interfacial structures, } \\
\text { strained layers, topological theory of defects }\end{array}$ \\
\hline Keywords (user supplied): & \multicolumn{2}{|c}{} \\
\hline
\end{tabular}

\section{S ScholaroNE" \\ Manuscript Central}




\title{
Strains and Rotations in Thin Deposited Films
}

\author{
J.P. HIRTH $\$$ and R.C. POND* \\ †114 E. Ramsey Canyon Rd., Hereford, AZ 85615, USA \\ *College of Engineering, Mathematics and Physical Sciences, Harrison Building, \\ University of Exeter, North Park Road, Exeter, EX4 4QF, U.K.
}

\begin{abstract}
Thin films deposited on misfitting substrates exhibit distortions produced by the superposition of coherency strains and the elastic fields of interfacial defects. These distortions become homogeneous strains, $\varepsilon$, and rotations, $\phi$, beyond a characteristic distance from the interface, $\mathrm{z}$, and are partitioned between the film and substrate. Residual strain arises when the density of interfacial defects is insufficient to compensate the intrinsic coherency strain, and is partitioned in a manner depending on the relative thicknesses of the two layers, d. However, rotations are not partitioned in this way. Expressions for the magnitude and partitioning of $\varepsilon$ and $\phi$ are derived for the case of elastically isotropic materials. Calculated values are shown to be in excellent agreement with experimental measurements for a variety of technologically relevant cases.
\end{abstract}

\section{Introduction}

Thin deposited films are widely exploited in modern technology. These films are often partially coherent, exhibiting arrays of dislocations at the interface between the misfitting film and its substrate. Such dislocations may accommodate coherency strains to some extent, but may also give rise to crystal rotations. Both these features affect the physical properties of a film, and a great deal of research has been directed

\footnotetext{
${ }^{*}$ Corresponding author. Email: r.c.pond@exeter.ac.uk
} 
at elucidating this. Understanding the role of dislocations in mitigating coherency strains was pioneered by Frank and van der Merwe in 1949 [1], and several reviews summarize the current position [2-5]. Rigid body rotations of hetero-epitaxial films away from the nominal orientation relationship were first reported by Igarishi in 1971 [6]: he attributed the rotations to dislocations in the interface with Burgers vector components inclined to the interface, thereby producing a small-angle tilt wall superposed on an underlying misfit array. Since that time numerous observations of rotated films have been reported and their consequences discussed [7-15].

This paper focuses on two aspects of coherency strains and rotations in a thin film, B, deposited on a substrate A: characteristic distances and partitioning. In the immediate vicinity of an interface (parallel to the plane $\mathrm{x}, \mathrm{y}$ ) the strain field has a complex form. However, at distances greater than $z^{m}$ any residual misfit strain (i.e. coherency strain uncompensated after the introduction of misfit dislocations) becomes constant. Moreover, these strains are partitioned along y into $\varepsilon_{y y}^{A}$ and $\varepsilon_{y y}^{B}$. (Superscripts A and B refer to-values of strain or rotation partitioned to the A and B crystals). Similarly, rotational distortions adopt constant values at distances greater than $z^{\phi}$, and are partitioned to the values $\phi^{A}$ and $\phi^{B}$. The characteristic distances $z^{m}$ and $z^{\phi}$, as well as the partitioning into $\varepsilon_{y y}^{A}$ and $\varepsilon_{y y}^{B}$ and $\phi^{A}$ and $\phi^{B}$, arise from the superposition of the elastic fields of the defects at the interface on the initial coherency strain in the film. Here, we compare the values of $z^{m}$ and $z^{\phi}$; in general these depend on the elastic properties of A and B [16], but we consider only the isotropic homogeneous case for illustration [17]. The partitioned values $\varepsilon_{y y}^{A}$ and $\varepsilon_{y y}^{B}$ are known to depend on the relative thicknesses of $\mathrm{A}$ and $\mathrm{B}, d^{A}$ and $d^{B}[18]$. However, in general, the partitioning into $\phi^{A}$ and $\phi^{B}$ does not depend on $d^{A}$ and $d^{B}$, as has become clear only recently from studies of interfaces in phase transformations [19,20]. Finally, we do not consider the plastic deformation that may lead to the interfaces we describe. Instead we deal with the properties of static interfaces once they have formed.

Coherent precipitates or martensite plates are subjected to biaxial stresses and strains arising from coherency. In addition, matrix compatibility constraints often lead to added stresses acting normal to the interface. However in the topological model [19, $20]$ or the closely related phenomenological theory of phase transformations [21, 22], 


\section{Interface Defects}

Evidently, the nature of defects at a particular interface is a key factor governing the resulting characteristic distances and partitioning. The two principal categories of line defects are dislocations and disconnections [23, 24]: the former are line-defects with Burgers vector $\mathbf{b}$, whereas the latter have dislocation and step character, $\mathbf{b}$ and $h$. Many observations of dislocations have been reported [25-30], some with $\mathbf{b}=\left[b_{x}, b_{y}, 0\right]$ parallel to the interface, and others with inclined $\mathbf{b}=\left[b_{x}, b_{y}, b_{z}\right]$. Figure 1(a) is a high-resolution TEM image showing the two types in an epitaxial interface between a deposited GaAs film and a Si substrate [31]. Figure 1(b) is a schematic illustration of the extra $\{111\}$ planes terminating at the cores of these dislocations [2]. Figure 1(c) is a schematic illustration of the formation of a disconnection when a surface step on a (0112) $\alpha-\mathrm{Al}_{2} \mathrm{O}_{3}$ substrate is overgrown by the incompatible surface step on a Si film [15]. The step height, $h$, is defined as the smaller of the two free surface step heights, $\mathrm{Si}$ in this case, and the $\mathbf{b}$ is equal to the difference between the translation vectors indicated in the figure. Accommodation of coherency strains and production of crystal rotations arise from arrays of defects, and three distinct situations are considered, as depicted in figure 2. Figure 2(a) depicts 


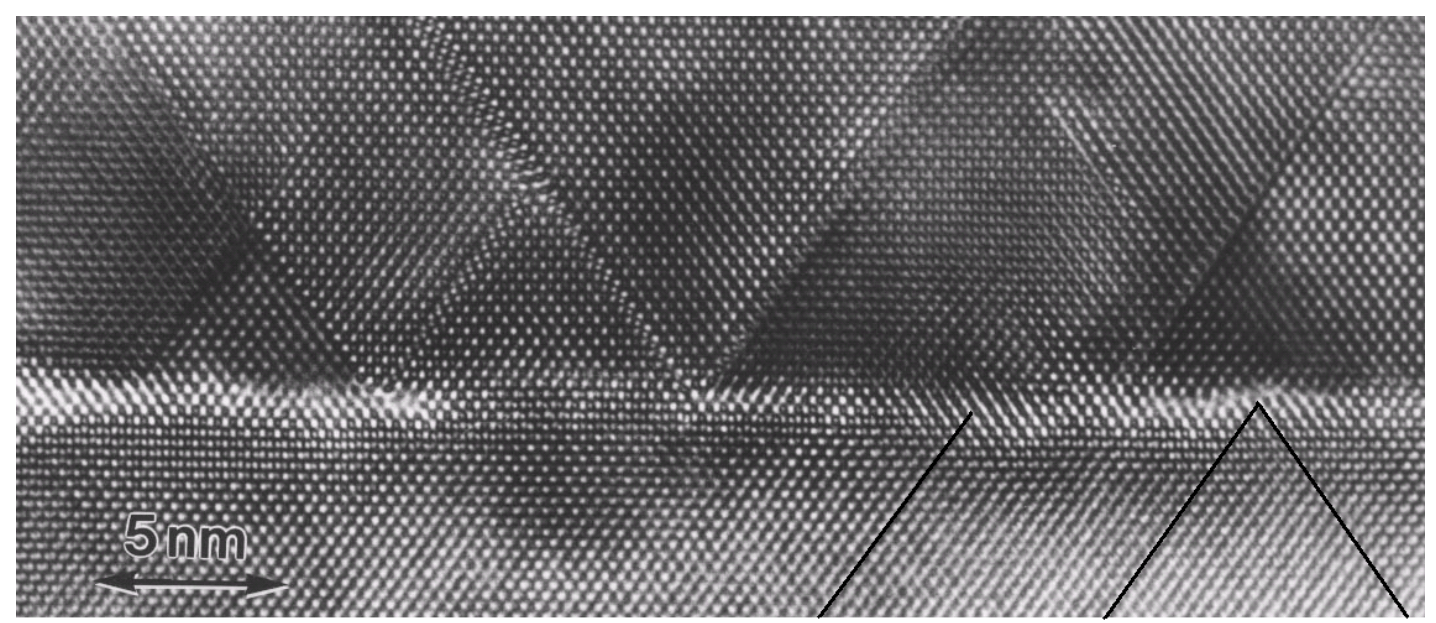

(a)

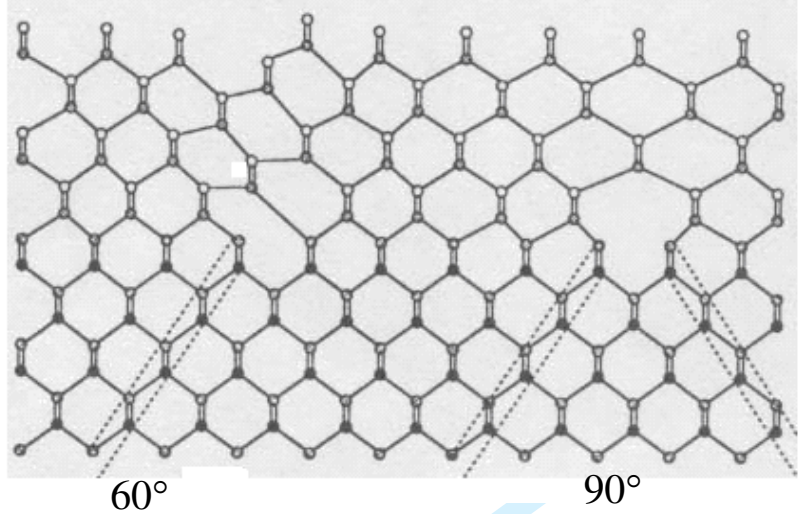

(b)

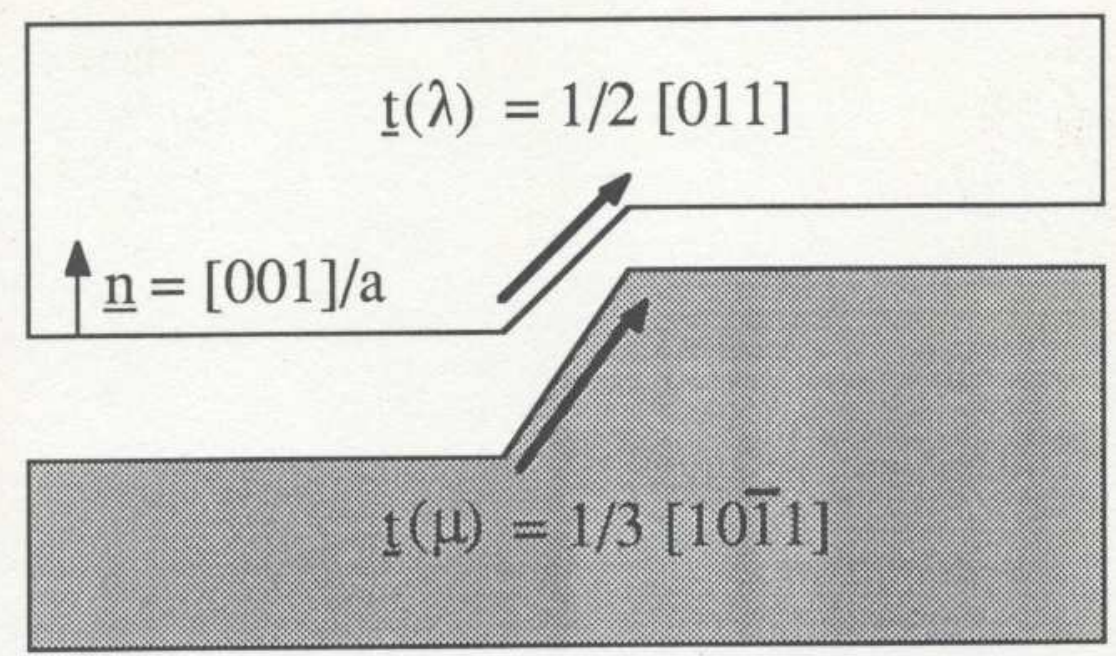

(c) 
a)

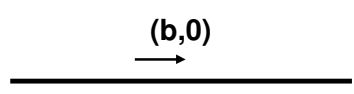

b)

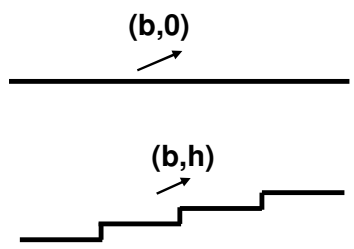

$\theta=0, \phi=0$

$$
\theta=0, \phi \neq 0
$$$$
\theta \neq 0, \phi \neq 0
$$

Figure 2 Schematic illustration of three principal interface topologies.

an interface where an array of dislocations with in-plane $\mathbf{b}$ accommodates misfit. Here, neither the substrate nor film surfaces are stepped, leading to an interface which remains parallel to $\mathrm{x}, \mathrm{y}$, designated $\theta=0$. Furthermore, since the dislocations exhibit no components $b_{z}$, no rotational distortions arise, hence $\phi=0$. In figure 2(b) the dislocations arrive at the interface after gliding through the film, B. The resulting interfacial dislocations have $\mathrm{h}=0$, but their finite components $b_{z}$ lead to rotations, hence $\theta=0, \phi \neq 0$. Figure 2(c) illustrates an array of disconnections: here the step array induces a rotation of the interface plane, and the finite $b_{z}$ produces a crystal rotation, i.e. $\theta \neq 0, \phi \neq 0$. An actual interface corresponds either to one of the three cases depicted in figure 2 , or to a combination that can be treated by superposition. 
For example, films are often deposited on vicinal substrate surfaces comprising an array of equally spaced steps. When overgrown, these steps may become disconnections, but this array is unlikely to fully accommodate the misfit. Consequently, additional dislocations may be introduced as in figure 2 (a) or (b). The characteristic distances and partitioning for these possibilities are addressed in sections 3.1 and 3.2 where elastic distortions with $\phi=0$ and $\phi \neq 0$ respectively are considered.

\section{Elastic distortions.}

\subsection{Misfit without rotation $(\phi=0)$.}

As an example of the simple misfit case, figure 2(a), we consider one parallel array of dislocations, with an attendant plane strain elastic field. Reference states for the definition of Burgers vectors are summarized in Appendix A. The general result for strain partitioning is given in Appendix B. For most purposes the simple engineering strain of equations (B5) and (B6), which also implies equal partitioning of the difference in lattice parameters of the two crystals in the small strain limit, suffices. The results for one array are qualitatively similar to the biaxial case with intersecting arrays: for the latter the form of Hooke's law differs and there are Poisson fields of one array superposed on the other [3, 32, 33, 34]. For an array of misfit dislocations with spacing $\lambda_{0}$, figure 3 (where the perfect crystal atom spacings in the y direction are $a^{A}>a^{B}$ ), the coherency strain can be envisioned as arising from a continuous distribution of infinitesimal dislocations. The integral of this distribution per unit length, $\ell$, is $\mathbf{b}_{y}^{c} / \ell$, and this is numerically equal to the total coherency strain, $\varepsilon_{y y}^{c}$ (see Appendix A). For the present case this representation of the distribution leads to no loss in rigor. The zero long-range strain condition is satisfied for the equilibrium spacing of misfit dislocations, $\lambda^{e}$. Then the Burgers vector of the misfit dislocations, $\mathbf{b}_{y}^{m}$, is equal to $-\mathbf{b}_{y}^{c}\left(\lambda^{e} / \ell\right)$, and $z^{m}=z^{e}$. 


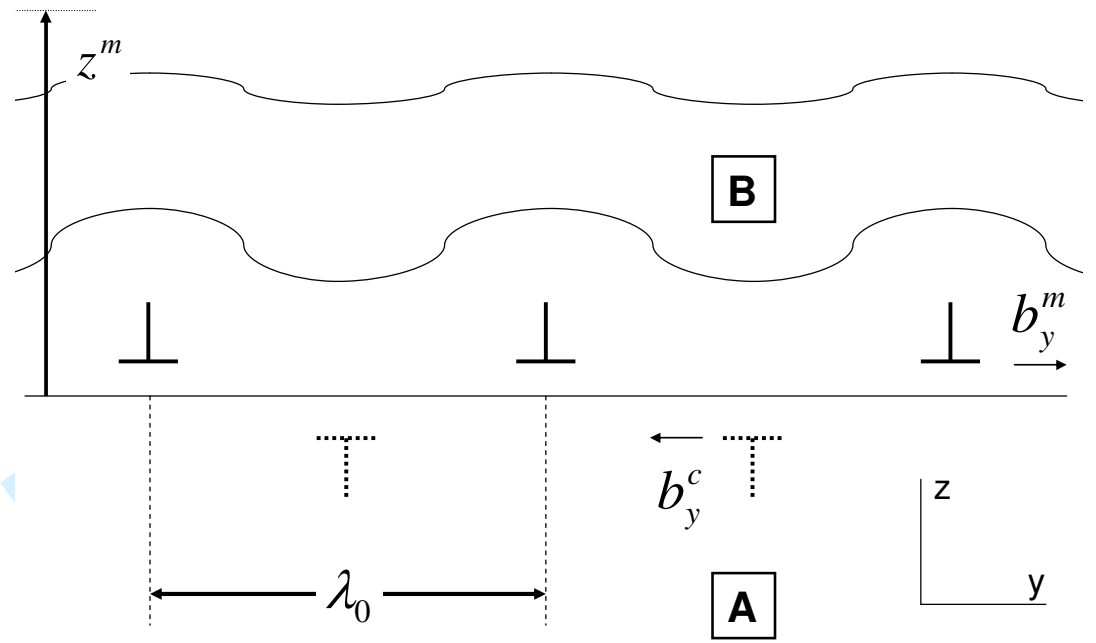

Figure 3. Misfit dislocations spaced by $\lambda_{0}$ at the interface between $\mathrm{A}$ and $\mathrm{B}$. The curved line represents a constant value of $\varepsilon_{y y}^{m}$; the strains tend to become constant for

$$
z>z^{m} \text {. }
$$

Near the misfit dislocations, $\mathrm{r}<<\lambda_{0}$, their elastic fields approach the $\ln r$ field present at a distance $\mathrm{r}$ from an isolated dislocation. For $\mathrm{r} \sim 0.1 \lambda_{0}$, the field becomes the hyperbolic sinh/cosh field of parallel arrays of dislocations [3, 33, 34]. For $z>z^{m} \approx \lambda_{0}$, the misfit dislocation field rapidly converges to a uniform strain state, $\varepsilon_{y y}^{m}=b^{m} / \lambda_{0}$, other $\varepsilon_{i j}^{m}=0$, in accord with St. Venant's principle. The zero longrange condition is defined when the misfit strain is equal and opposite to the interface coherency strain $\varepsilon_{y y}^{c}=-\Delta a /\langle a\rangle$, where $\Delta a=a^{A}-a^{B}$ and $\langle a\rangle=\left(a^{A}+a^{B}\right) / 2$. At free surfaces normal to $y$, there are also varying surface forces and torques to satisfy the free surface boundary condition, but the accompanying strains and twists are confined to a distance $\approx \lambda_{0}$ from the free surface [35] and need not be considered here. Beyond this distance, image strains become uniform. For a thin bilayer, these lateral strains would produce bending in a free bilayer. These are eliminated for a thin central member of a trilayer, or a thin layer in a multilayer.

Figure 4 is a plot of $\varepsilon_{y y}^{m}$ as a function of $\mathrm{z}$ on the same size scale as figure 3. The figure indicates the uniformity of strain for $z>z^{m}$. For figure 4(a), the case of an equal layer thickness bicrystal $d^{A}=d^{B}$, the Newtonian forces must vanish on a plane 
normal to $y$, so that both the misfit and coherency strains are equally partitioned to the two layers. When there is no long-range strain, $\varepsilon_{y y}^{m B}=\varepsilon_{y y}^{m} / 2=-\varepsilon_{y y}^{c B}=-\varepsilon_{y y}^{c} / 2$ and $\varepsilon_{y y}^{m A}=-\varepsilon_{y y}^{m} / 2=-\varepsilon_{y y}^{c A}=\varepsilon_{y y}^{c} / 2$. The condition $\varepsilon_{y y}^{m}=-\varepsilon_{y y}^{c}$ is the same whether there is a factor $1 / 2$ on both sides or not, so the basic equivalence of misfit and coherency strain is the same whether one deals with the partitioned strains or the interface condition. For figure 4(b), the force balance leads to the partitioning of long-range coherency strains in the form

$$
\varepsilon_{y y}^{c A}=\varepsilon_{y y}^{c} d^{B} /\left(d^{A}+d^{B}\right)
$$

and

$$
\varepsilon_{y y}^{c B}=\varepsilon_{y y}^{c} d^{A} /\left(d^{A}+d^{B}\right) .
$$

Similar expressions can be written for $\varepsilon_{y y}^{m A}$ and $\varepsilon_{y y}^{m B}$. The strains now lie mainly in the thin film. The characteristic distance for this partitioning is the smaller of the layer thicknesses, here $d^{B}$. Usually, the characteristic distance for strain partitioning, $d^{B}$, greatly exceeds that for uniformity of strain, $z^{m}$. For the relaxed, zero long-range strain case, the dislocation strains are equal and opposite to the coherency strains when $z>z^{m}=z^{e}$, and are also of the form in figure 4 .
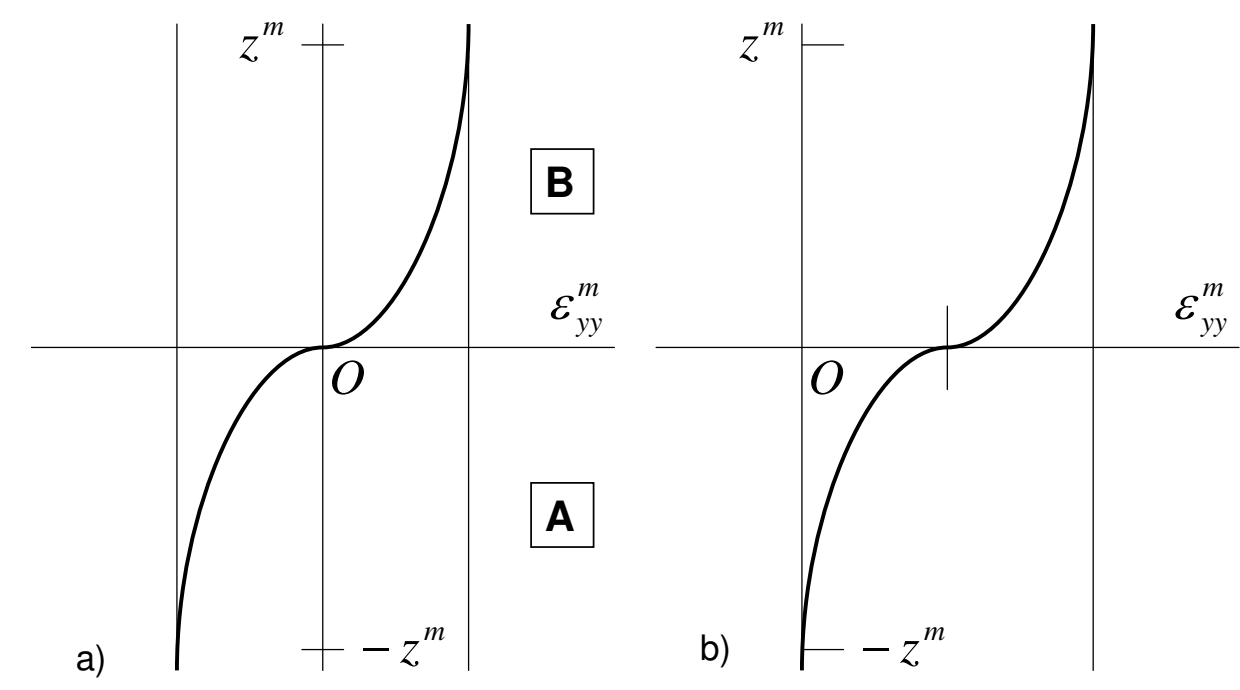
Figure 4. Strain $\varepsilon_{y y}^{m}$ of misfit dislocation array as a function of $\mathrm{z}$ for a fixed value of $\mathrm{y}$. The sign and magnitude of $\varepsilon_{y y}^{m}$ vary with y but $z^{m}$ remains nominally the same. a) equal layer thickness case, $d_{A}=d_{B}$. b) Thin film case, $d=d_{B}<<d_{A}$.

3.2 Misfit with rotation $(\phi \neq 0)$.

For the inclined dislocation case, the misfit dislocations deposited on the interface have both $b_{y}^{m}$ and $b_{z}^{m}$ components, figure 5. To first order, the $b_{y}^{m}$ component follows the above analysis, cancelling $b_{y}^{c}$ for the zero long-range strain condition. The field of the tilt wall can then be considered to superpose on the misfit dislocation array, producing a rotation of the two crystals by an angle $\phi$. To nonlinear order, the presence of the tilt wall changes the length $\lambda_{0}$ for the analysis of sect. 3.1 to $\lambda=\lambda_{0} / \cos (\phi / 2)$ [36], figure 6. The misfit condition is expressed in the rotated $x_{i}{ }^{\prime}$ coordinates: $b_{y}{ }^{\prime} / \lambda=-\varepsilon_{y y}^{c}{ }^{\prime}$. Actually both $b_{y}{ }^{\prime} / \lambda$ and $\varepsilon_{y y}^{c}{ }^{\prime}$ are partitioned to the two crystals, but the resulting factors of $1 / 2$ cancel. Here $b_{y}{ }^{\prime}=b_{y} \cos (\phi / 2)-b_{z} \sin (\phi / 2)$ and $\varepsilon_{y y}^{c}{ }^{\prime}=\varepsilon_{y y}^{c} \cos ^{2}(\phi / 2)$. Poisson terms of order $\sin ^{2}(\phi / 2)$ are completely negligible and nearly cancel for the relaxed arrangement anyway. Hence the misfit spacing that relieves long range stress is

$$
-\varepsilon_{y y}^{c}=\left[b_{y}-b_{z} \tan (\phi / 2)\right] / \lambda_{0} \approx b_{y} / \lambda_{0} .
$$

The angle $\phi$ is given by the Frank relation

$$
\phi=2 \sin ^{-1}\left(b_{z} / 2 \lambda_{0}\right) \approx b_{z} / \lambda_{0}
$$

where the approximate forms are accurate in the limit of small $\phi$. Analogous to the nonlinear treatment of phase transformations [36], equations (2) and (3) are solved for $\lambda_{0}$ and $\phi$ by iteration. $\lambda_{0}$ is estimated from the approximate form in equation (2) and $\phi$ is determined from the exact (3). This value of $\phi$ is substituted in the exact (2) to give a new $\lambda_{0}$ and iteration proceeds. The convergence is rapid because of the weak 
dependence of $\phi$ on $\lambda_{0}$. As shown explicitly in Appendix C, the rotations partition to $\pm \phi / 2$ in the two crystals.

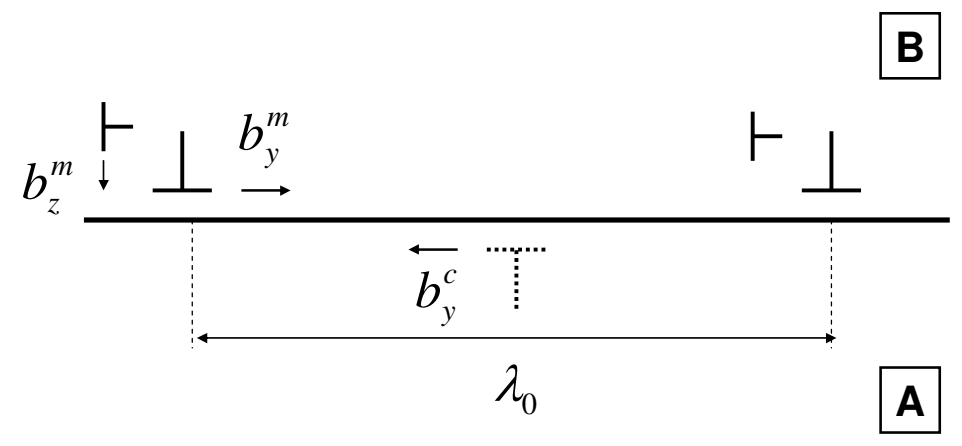

Figure 5. Crystal dislocations impinge on the interface to relieve misfit. Their Burgers vectors are inclined to the interface, having both misfit, $b_{y}^{m}$, and tilt, $b_{z}^{m}$, components.

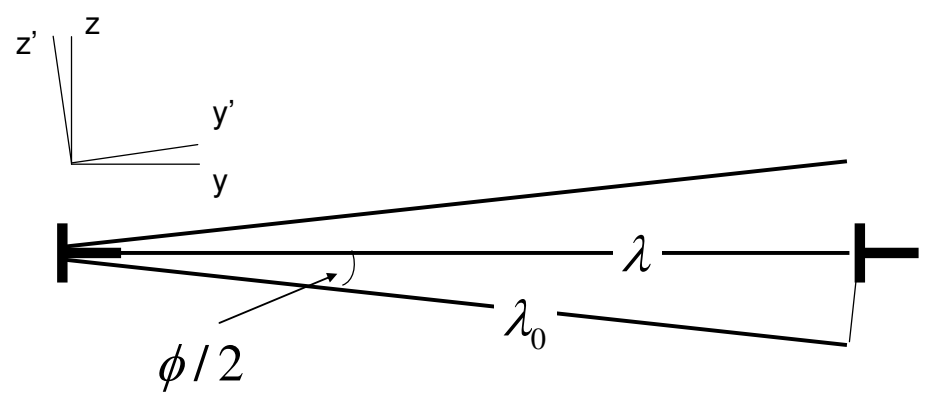

Figure 6. A tilt wall rotates the terrace plane by $\pm \phi / 2$ and changes the dislocation spacing from $\lambda_{0}$ to $\lambda=\lambda_{0} \cos (\phi / 2)$. The coordinates $x^{\prime} y^{\prime} z^{\prime}$ are selected to be consistent with figure 1.

As shown in equation C-5 in Appendix $\mathrm{C}$ and as depicted in figure 7 there are local distortion fields $\partial u_{i} / \partial x_{j}$, near the dislocations, where the $u_{i}$ are displacements. These comprise both strains, $\left[\partial u_{i} / \partial x_{j}+\partial u_{j} / \partial x_{i}\right] / 2$, and rotations, $\left[\partial u_{i} / \partial x_{j}-\partial u_{j} / \partial x_{i}\right] / 2$. As indicated in figure 4 , the strains rapidly converge to zero in the fully misfit relieved case for $z>z^{e}$, leaving only the rotations at long-range. For $z>z^{e}$ the strains vanish, so force equilibrium is satisfied. Hence, unlike the misfit 
strains, which depend on the ratio $d_{B} / d_{A}$, the rotations partition equally to the two crystals [37], which each have the planes corresponding to the coherent terrace planes rotated by $\phi / 2$ relative to the interface. A similar result would apply for a layer imbedded in a multilayer or a thin central member of a trilayer. With any bending subtracted, it would also apply for a bilayer thin film.

$z$

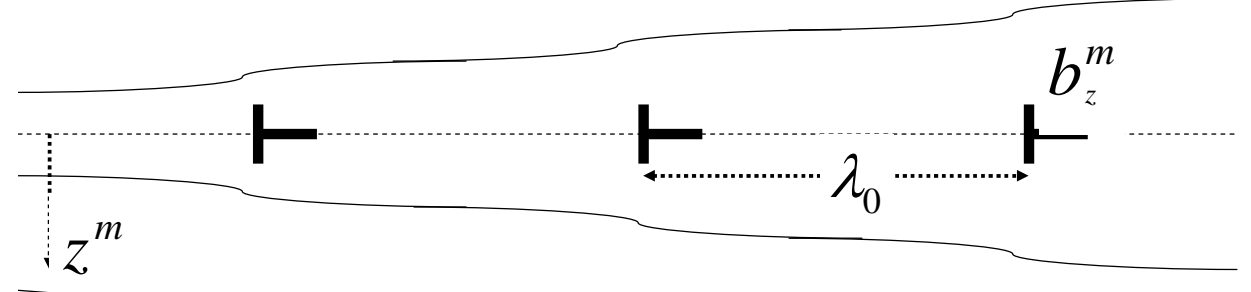

Figure 7. The local distortions of a tilt wall are represented by the curved lines. For $z>z^{m}$ these distortions rapidly converge to pure rotations, partitioned to $\phi / 2$ in each crystal.

\subsection{Misfit accommodation by disconnections $(\theta \neq 0, \phi \neq 0)$.}

For the offcut disconnection case, figure 2(c), the nucleation mechanism [38, 39] tends to favor the incorporation of a large misfit component in the disconnection, as in figure $1(\mathrm{c})$, in turn leading to a component $b_{z}{ }^{\prime}$ in the habit plane. For the special case of cube on cube deposition, the component $b_{z}$ ' can be very small [40]: we do not consider such a case here. For the offcut case or when some like-sign disconnections occur in a local region during growth, when $b_{z}{ }^{\prime} \neq 0$, the imposed disconnection density may not completely remove misfit and added misfit dislocations might be needed.

We first consider the disconnections, depicted in figure 8 . The added feature is that the step height $\mathrm{h}$ equals the smaller of the complementary free-surface steps and not necessarily the vicinal step height. The steps cause an interface rotation, $\theta$. Now, the misfit balance and the tilt rotations are performed in the rotated coordinates $x_{i}{ }^{\prime}$ as 
shown in the figure. A nonlinear treatment, analogous to that for misfit dislocations, is available for the disconnection case [36]. The equilibrium disconnection spacing that produces a long-range dislocation strain equal and opposite to the coherency strain is

$$
-\varepsilon_{y y}^{c}=\frac{\left[b_{y}+b_{z} \tan (\theta-\phi / 2)\right] \tan \theta}{h}=\frac{\left[b_{y}+b_{z} \tan (\theta-\phi / 2]\right.}{\lambda_{0}} \approx \frac{\left[b_{y}+b_{z} \tan \theta\right]}{\lambda_{0}}
$$

The angle $\varphi$ is given by

$$
\begin{gathered}
\phi=2 \sin ^{-1}\left\{\frac{\left[b_{z} \cos (\theta-\phi / 2)-b_{y} \sin (\theta-\phi / 2)\right] \cos (\theta-\phi / 2) \tan \theta}{2 h}-\frac{\varepsilon_{y y}^{c} \sin (\theta-\phi / 2) \cos (\theta-\phi / 2)}{2}\right\} \\
\cong 2 \sin ^{-1}\left\{\frac{\left[b_{z} \cos \theta-b_{y} \sin \theta\right] \sin \theta}{2 h}-\frac{\varepsilon_{y y}^{c} \sin \theta \cos \theta}{2}\right\} \approx \frac{b_{z}}{\lambda_{0}}
\end{gathered}
$$

Here, the successive approximations become accurate in the limit of small $\phi$ and small $\theta$, respectively. Evidently, when $\theta$ is zero, equations (4) and (5) reduce to (2) and (3), respectively. Just as for the misfit dislocation array cases (figure $2 a$ and $b$ ), the lattice rotations in the disconnection case partition equally when $z>z^{e}$, in accord with equation C-5 in Appendix C, and there is no dependence on the ratio $d_{B} / d_{A}$. When $\lambda_{0}$ is increasingly large, the angle $\phi$ becomes so small as to be negligible.

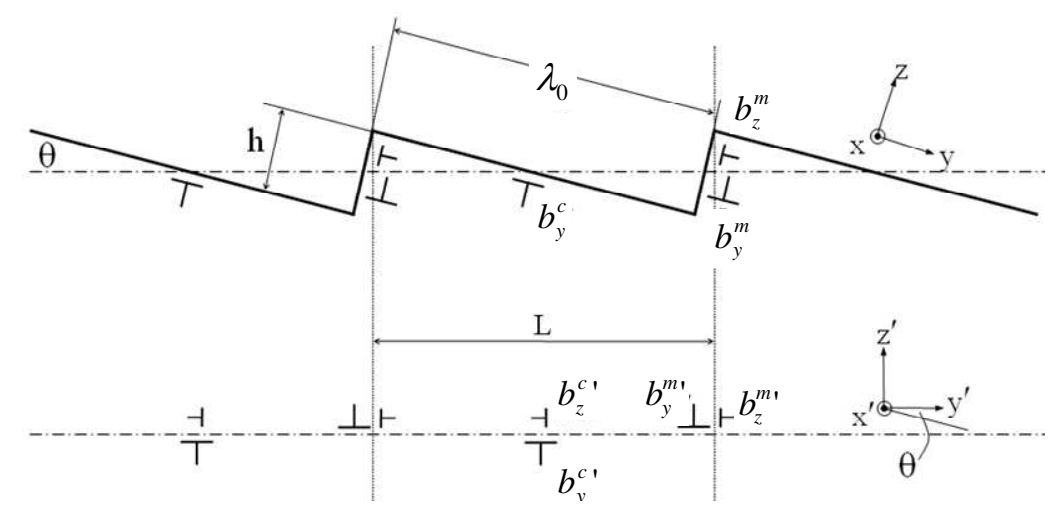

Figure 8 Schematic illustration showing the disconnection content of an interface, with Burgers vector components resolved in the terrace (upper) and habit plane (lower) frames [19, 20 ]. The terrace plane is inclined at an angle $\theta$ to the horizontal 
In general the disconnections from vicinal steps do not remove all the coherency strain. The disconnection spacing $\lambda_{0}$ is known so equation (2) gives the portion of the strain removed (or added), $\varepsilon_{y y}^{c v}$. The difference between this value and $\varepsilon_{y y}^{c}$ is the portion of the strain to be removed by misfit dislocations, either in-plane or inclined. The nominal spacing of these defects can be determined from equation (2) and (4) in the usual manner. Actually, while the strain portions are known, and hence the numbers of the two types of defects, the spacings $\lambda_{0}$ do not remain constant. The spacings rearrange in a somewhat nonuniform manner so that the total strain energy of the defects is minimized. The consequence is that the $z^{m}$ distance where the strain becomes uniform is somewhat smaller than the smaller of the two individual uniform spacings. There could be a similar mixture of the two types of misfit dislocations, although in that case only the dislocations with in-plane Burgers vectors would rearrange to minimize strain energy: The inclined dislocations would be sessile for inplane motion. The determination of the effective value of $z^{\phi}$ for such superposed arrays becomes more complicated.

The convergence of the strains to zero is less rapid, reflecting the variation in spacings of the two types of defects, but is still of the order of the average spacing of the defects. Hence, $z^{\phi}$ will still be much less than the characteristic distance, say $d^{B}$, for the superposed arrays. In principle, the disconnections could even increase misfit strain, although this is not commonly expected. The major role of the vicinal steps is to augment island (disc or spherical segment) nucleation there during deposition [38, 39]. Elastic energy has a role (not considered in detail in the original papers) but surface energy is a major factor. The latter factor may dominate, allowing the unfavorable elastic energy nucleus to form.

Hence, there typically should be no effect of relative layer thicknesses on the partitioning of rotations, provided that the layer thickness $d^{B}>z^{\phi}$. Other effects, such as differing elastic constants, would partition rotations at the larger size scale, 
analogous to the misfit strains considered here, but these effects vanish in the isotropic elastic case. When $d^{B}<z^{\phi}$, i.e. for widely spaced misfit defects (inclined dislocations or disconnections), the local rotations become a complicated function of position. The net rotation may be less than in the large $\mathrm{z}$ limit. Of course the net rotation becomes small when the misfit defects are widely spaced, so this limit does not represent a very important case.

\section{Applications}

One example of each of the three interface types in figure 2 and one case of superposed types (a) and (c) are discussed in this section. In each example, no long range coherency strain persists, i.e. $\varepsilon_{y y}^{c}+\varepsilon_{y y}^{m}=0$. We focus on the characteristic distances $z^{m}$ and $z^{\phi}$ : in the first example only $z^{m}$ is meaningful: in the second and third examples $z^{m}=z^{\phi}$ : in the fourth example, $z^{m} \neq z^{\phi}$.

4.1 Misfit dislocation array with in-plane $\mathbf{b}: \theta=0, \phi=0$

An example of this case is the growth of (001)GaAs on (001) Si substrates, as illustrated in figure1(a) from the work of Xing et al. [31]. The lattice parameters for these crystals at room temperature are $0.5653 \mathrm{~nm}$ and $0.5431 \mathrm{~nm}$ respectively, giving $\varepsilon_{y y}^{c}=\varepsilon_{x x}^{c}=\varepsilon^{c}=-0.0401$. An orthogonal array of $1 / 2<110>$ edge dislocations with spacing $\lambda^{e}=b_{y}^{m} / \varepsilon_{y y}^{c}=9.7656 n m$ would accommodate this misfit. Using weakbeam TEM, Eaglesham et al. [41] have observed defect arrays consistent with this proposition as shown in figure 9 , although $60^{\circ} 1 / 2<101>$ dislocations were also found to be present. Thus, $z^{c} \approx \lambda^{e}$ is about $10 \mathrm{~nm}$ in this high misfit case. No tilting is anticipated (provided the $b_{z}$ components of the $60^{\circ}$ dislocations sum to zero).

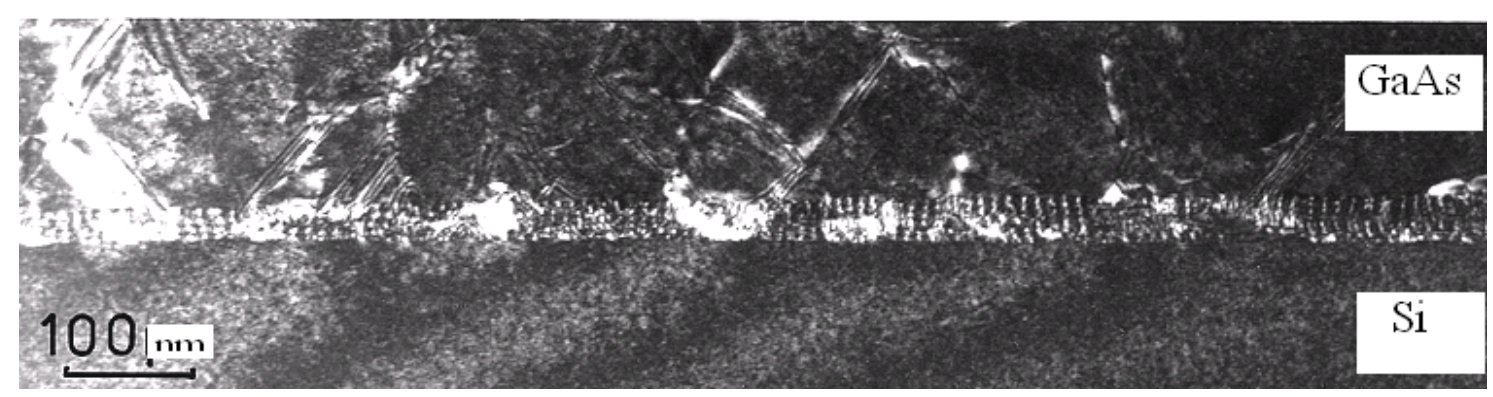


Figure 9. Weak beam TEM image showing one array of an orthogonal network of $90^{\circ}$ $1 / 2<110>$ dislocations in (001) GaAs:Si [31].

4.2 Misfit dislocation array with out-of-plane $\mathbf{b}: \theta=0, \phi \neq 0$ :

Du and Flynn used MBE to grow epitaxial films of hcp and fcc materials on bcc substrates [14]. Very thin films were observed by X-ray diffraction to be homogeneously strained to be commensurate with the substrate, but this coherency strain was relieved in thicker deposits. The thicker films were also tilted by values up to several degrees: for example, (110) films of $\mathrm{Cu}$ grown on (211) substrates of Mo were tilted by $7.34^{\circ} \pm 0.1^{\circ}$ about $[1 \overline{10}]_{C u}$. This observation can be understood in terms of misfit relief along $[001]_{C u} /[01 \overline{1}]_{M o}$ by equal numbers of $1 / 2[\overline{101}]_{C u}$ and $1 / 2[0 \overline{11}]_{C u}$ dislocations gliding on (111) $)_{C u}$ [42]. These defects would lie in the interface along $y / /[001]_{C u}$, with zero overall screw component, $b_{x}$, where $x / /[110]_{C u}$. Their identical $b_{z}=1 / 4[110]_{C u}$ components produce the tilting. The lattice parameters for $\mathrm{Cu}$ and Mo are $0.3615 \mathrm{~nm}$ and $0.3147 \mathrm{~nm}$ respectively, giving the extremely large misfit $\varepsilon_{y y}^{c}=\ln \left(1-\Delta a / \sqrt{2} a_{M o}\right)=-0.2079$ (c.f. $\Delta a /<a>=0.2072$ ). The Burgers vector components are $b_{y}^{m}=a^{i} / 2=0.2006 n m$, and $b_{z}^{m}=-\sqrt{2} a_{C u} / 4=-0.1278 n m$, so the approximate forms of equations (2) and (3) give $\lambda_{0}=0.9649 \mathrm{~nm}$ and $\phi=7.5889^{\circ}$ respectively. With the iteration procedure described in section 3.1 these values change to $(1.0057 \mathrm{~nm}, 1.004 \mathrm{~nm}, 1.0041 \mathrm{~nm})$ and $\left(-7.2860^{\circ},-7.2979^{\circ},-7.2975^{\circ}\right)$, where successive iterations are listed in sequence. This rotation of the deposited film by $7.30^{\circ}$ is clockwise about $[1 \overline{10}]_{C u}$ and is near the centre of the experimental accuracy range. Thus, $z^{m}$ and $z^{\phi}$ are equal to about $1 \mathrm{~nm}$ in this case.

Relevant to the present analysis, when inclined dislocations are present they tend to group in areas where only one Burgers vector is present among the possible variants. This would give another size scale in the strain/rotation partitioning. However, in crystals of lower symmetry or for interfaces of lower symmetry, there may be only one possible inclined-type dislocation. The same methodology as described here would apply to thin films formed by a decomposition to form pearlite 
or to thin plates formed as in situ composites by solidification or by heavy deformation as for wire drawing [43].

\subsection{Misfit disconnection array: $\theta \neq 0, \phi \neq 0$}

Disconnection arrays have been observed using TEM in parent-martensite interfaces $[19,20]$. In this case, the disconnections are mobile, and form arrays as in figure 8 with spacings close to the equilibrium value, $\lambda^{e}$. (In general, a second array of dislocations, inclined to the disconnection array, is present in the interface for complete misfit accommodation). Figure 10 shows a disconnection array in Ti$10 \mathrm{wt} . \%$ Mo [44], and we compute the value of $\theta$ and $\phi$ using equations (4) and (5). Equation. (4) can be written

$$
\left[b_{y} \tan \left(\phi_{i} / 2\right)+b_{z}\right] \tan ^{2} \theta_{i+1}+\left[\left(h \varepsilon_{y y}^{c}-b_{z}\right) \tan \left(\phi_{i} / 2\right)+b_{y}\right] \tan \theta_{i+1}+h \varepsilon_{y y}^{c}=0 .
$$

One can start with the linear result $\phi_{i}=\phi_{1}$, and subsequently solve expression (6) for the up-dated $\theta_{i+1}$. Then the updated $\phi_{i+1}$ can be found using eq. (5) in the form $\phi_{i+1}=f\left(\theta_{i+1}, \phi_{i}\right)$. Convergence is rapid since $\phi_{i+1}$ depends only weakly on $\phi_{i}$.

For this example $\mathrm{h}_{\alpha}=0.2556 \mathrm{~nm}, \mathrm{~h}_{\beta}=0.2678 \mathrm{~nm}$ and $\varepsilon_{y y}^{c}=0.038$ (the strain $\varepsilon_{x x}^{c}=0.0086$ has been neglected for simplicity). The values of $h_{\beta} / h_{\alpha}, \theta, \phi, \omega_{\alpha}, \omega_{\beta}$ are shown in the Table. Evidently, $\phi$ is small in this case and the approximate (linear) forms of equations (4) and (5) are satisfactory. In fact, this is the case for most martensitic transformations (which involve modest volume changes). The calculated values are consistent with experimental observations, though the uncertainties in the latter are of the order $\pm 1^{\circ}$ and $\pm 0.25^{\circ}$ for $\omega$ and $\phi$ respectively in ideal conditions. Only in exceptional cases would the non-linear corrections be needed; an example would be the martensitic transformation in a hypothetical Ti alloy with the same value of $\varepsilon_{y y}^{c}$ as above but with $h_{\beta} / h_{\alpha}=1.15$, as shown in the Table. A further real example arises in Pu-Ga alloys, where $\phi \approx 6^{\circ}$ and $h_{\beta} / h_{\alpha}=1.2$ [45]. The rotation produced in 


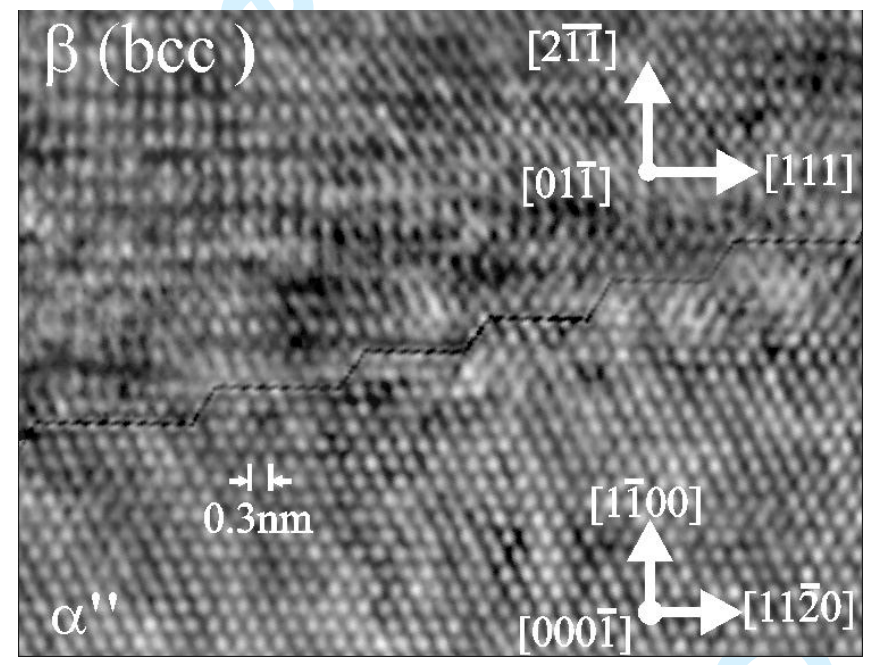

Figure 10. Transmission electron micrograph of a parent-martensite interface in Ti$10 \mathrm{wt} . \% \mathrm{Mo}$ [44]. The electron beam is oriented parallel to the disconnection array, as in figure 8 .

Table: Step height ratios and habit plane parameters for "Ti" obtained by the linear and non-linear forms of equations (4) and (5). All angles are expressed in degrees.

\begin{tabular}{|l|l|l|l|l|l|}
\hline & $h_{\beta} / h_{\alpha}$ & $\theta$ & $\phi$ & $\omega_{\alpha}$ & $\omega_{\beta}$ \\
\hline
\end{tabular}




\begin{tabular}{|c|c|c|c|c|c|}
\hline & & & & & \\
\hline Linear & 1.048 & 10.8609 & 0.5286 & 10.5966 & 11.1252 \\
Non-Linear & & 10.8727 & 0.5292 & 10.6080 & 11.1373 \\
\hline Linear & 1.15 & 10.0091 & 1.5169 & 9.2507 & 10.7676 \\
Non-Linear & & 10.0934 & 1.5299 & 9.3284 & 10.8583 \\
\hline
\end{tabular}

A special case occurs for some offcut crystals where disconnections form at the former free surface steps on the substrate, but no added misfit dislocations form. Hence the coherency strain is not completely relaxed. The above derivations are still applicable for this case, but $\lambda$ replaces $\lambda_{0}$ in equation (4) and the corresponding strain is only a portion of the equilibrium strain that would completely remove misfit.

4.4 Misfit dislocation array and superposed disconnection array: $\theta \neq 0, \phi \neq 0$

Aindow and Pond [15] studied the variation of tilting for (111) CdTe and (001) Si films on (0001) and (0112) $\mathrm{Al}_{2} \mathrm{O}_{3}$ substrates, respectively. Substrates were cut with a range of vicinal angles, $\phi^{v}$, and the resulting tilt of the deposited layer measured by Xray diffraction. The observed tilts were found to be consistent with the formation of disconnections, as illustrated for the $\mathrm{Si}: \mathrm{Al}_{2} \mathrm{O}_{3}$ case in figure 1(c). For the range of vicinal angles investigated, these defects are widely spaced, and therefore contribute minimally to the accommodation of misfit. In the $\mathrm{Si}: \mathrm{Al}_{2} \mathrm{O}_{3}$ case, misfit was accommodated by a non-orthogonal network of 1/2[110] and1/2[110] dislocations with spacings about $4 \mathrm{~nm}$, as shown in figure 11 [48]. Since their Burgers vectors are parallel to 


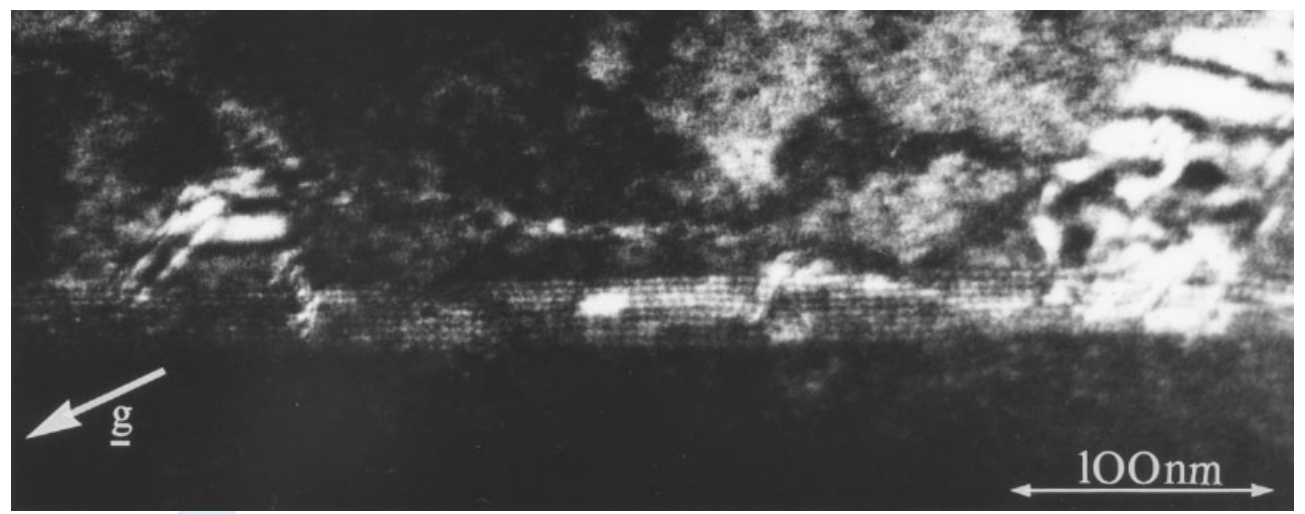

Figure 11. Weak beam TEM image of misfit dislocation array in (001) Si deposited on (0112) $\mathrm{Al}_{2} \mathrm{O}_{3}[48]$.

the substrate terrace planes, they do not contribute to film tilting in the first approximation. The CdTe: $\mathrm{Al}_{2} \mathrm{O}_{3}$ case is similar, although the misfit dislocation array (thought to be a triangular network of in-plane $1 / 2<1 \overline{10}>_{C d T e}$ dislocations) was not imaged directly. Thus, the observed tilts, $\phi$, are produced by the $b_{z}$ components of the disconnection array, and Aindow and Pond [15] correlated these with measurements of the vicinal angle of the substrates, figure 12. Vicinal angles can be expressed as $\phi^{v}=-h^{A} / \lambda^{v}$, where $\lambda^{v}$ is the spacing of the vicinal steps (the minus sign is necessary for consistency with the sense of $\phi$ defined in Appendix C), and $h^{A}$ is the height of the substrate surface steps. Since $\phi \approx b_{z} / \lambda^{v}=\left(h^{B}-h^{A}\right) / \lambda^{v}$, we can write $\phi \approx\left(1-h^{B} / h^{A}\right) \phi^{v}$. Thus the slope of a $\phi$-versus- $\phi^{v}$ plot is negative when $h^{B}>h^{A}$ and positive otherwise. The dashed lines in figure 12 represent the ideal relation associated with particular disconnections, and these show excellent agreement with the least squares fit to the data, solid line. 


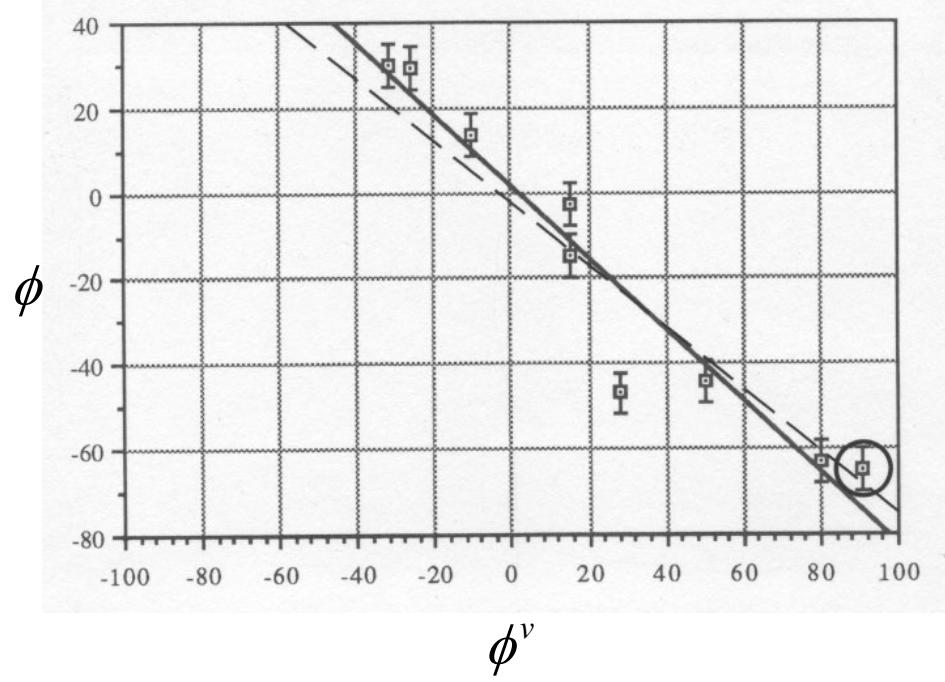

(a)

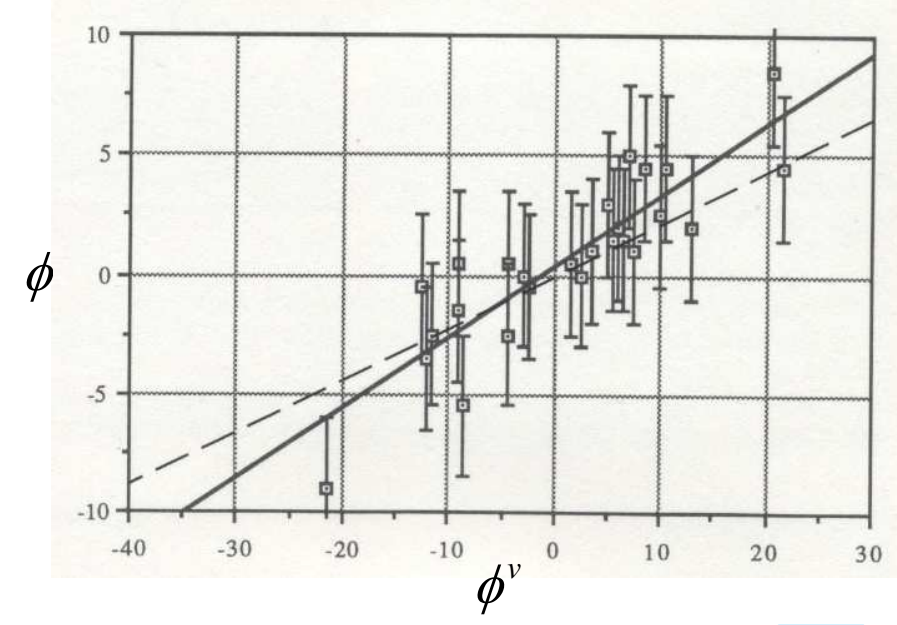

(b)

Figure 12. Correlation of film tilt angle, $\phi$, with surface vicinal angle, $\phi^{v}$, for (a) (111) CdTe on (0001) $\mathrm{Al}_{2} \mathrm{O}_{3}$ and (b) (001) $\mathrm{Si}$ on (0112) $\mathrm{Al}_{2} \mathrm{O}_{3}$. All angles are in units of minutes of arc. Solid lines represent the least-squares best-fit to the data, and the dashed lines are the ideal relations for specific disconnections [15].

The co-existence of a misfit dislocation array and an array of disconnections implies that $z^{m}$ and $z^{\phi}$ are not equal. In the Si case for example, $z^{m} \approx \lambda^{e} \approx 4 n m$, whereas 


\section{Discussion}

In the foregoing we have identified the characteristic lengths arising from interfacial dislocation and disconnection arrays. When residual strain persists the elastic misfit and coherency strains are partitioned according to equation (1), the characteristic distance for this partitioning being the smaller of the layer thicknesses, here $d^{B}$. For the relaxed, zero long-range strain case, the two strains are equal and opposite when $z>z^{m}=z^{e}$. In this case $z^{m}=z^{\phi}$ for all three interface types illustrated in figure 2. For a "mixed" case, such as (001) $\mathrm{Si}$ on (0112) $\mathrm{Al}_{2} \mathrm{O}_{3}, z^{m}$ is determined primarily by misfit dislocation spacing, and $z^{\phi}$ by the vicinal step spacing, and $z^{\phi}>z^{m}$. The strain partitioning depends on $\mathrm{d}^{\mathrm{B}}$ but the rotation partitioning is independent of $\mathrm{d}^{\mathrm{B}}$ provided that $\mathrm{d}^{\mathrm{B}}>\mathrm{z}^{\mathrm{m}} \approx \mathrm{z}^{\varphi} \approx \lambda_{0}$.

We have presented the above analysis for the plane strain, single-array case. However the characteristic lengths, the focus of the present treatment, would be the same for the biaxial strain, intersecting-array case as well. The characteristic lengths would also be similar for an imbedded layer or for a layer within a multilayer. The results also have implications for the phase transformation case. For lath type transformation products or for plate-shaped products with large aspect ratios, the rotations produced by interface defects should also be fully partitioned when the plate thickness is greater than the defect spacing in the interface.

While the presence of rotations associated with tilt components of dislocations and disconnections have long been known, the present results provide explicit results 
for expected rotations and their partitioning. Particularly significant is the finding that the critical length scale for achievement of long-range rotation partitioning is distinct from that characterizing the partitioning of residual long-range elastic strains. From a practical point of view, the dislocation and disconnection sites should provide local charged defect centers that can act as traps for electrons, holes and ionic defects such as vacancies while the inhomogeneous strain fields also give weak scattering of the electronic defects. The disconnections have smaller Burgers vectors than the misfit dislocations in general and thus represent weaker trapping sites, with concordant implications for electronic properties.

However, the disconnections and inclined-b dislocations with a misfit component carry rotations with them, also giving electron scattering. Indeed the deleterious effect of tilt wall components and accompanying rotations has been demonstrated for a high $\mathrm{T}_{\mathrm{c}}$ superconductor [49]. Understanding the source and magnitudes of the rotations provides an opportunity in thin film design.

As an example of a method to control the degree of rotation, research on films of $\mathrm{Ni}$ on $\mathrm{Cu}$ agree with the above trends, but with differences in whether the observed misfit dislocations are Lomer dislocations with $1 / 2<110>$ Burgers vectors lying in the interface $[25,27,28]$ or with in-plane and out-of-plane Burgers vectors inclined to the interface [26, 29]. Recent work [50] has shown that near the critical thickness, the dislocations are mainly inclined dislocations of this type and that with increasing thickness these convert increasingly to Lomer dislocations. Consistent with this finding, in-plane bs predominate in high-misfit cases: e.g. they form at island edges, whereas out-of-plane bs arise in 2-D growth films where misfit is lower [2]. Thus selecting the degree of misfit is a way to control the degree of rotation at an interface in such systems.

Both the nonlinear pair of equations (4) and (5), derived from the topological model for phase transformations [36], and equations (2) and (3) for the misfit case involve simultaneous transcendental equations that must be solved by iteration. Yet in many cases, the linear forms of the equations can be used. Then $\varphi$ in equations (3) and (5) is linearly related to $1 / \lambda_{0}$ and after substitution into equations (2) and (4), the latter can be solved directly for $\lambda_{0}$. This is the basis of the initial linear model for the topological model $[19,20]$, where for the phase transformation case one first solves 


\section{Summary}

The strains and rotations arising in thin films deposited on misfitting and/or offcut substrates have been quantified for the case of elastically isotropic materials. These distortions become homogeneous at distances greater than a characteristic distance related to the spacing of interfacial defects, $z^{m} \approx \lambda_{0}$. Strains are partitioned in a manner dependent on the ratio $d^{B} / d^{A}$ when the interfacial defects present do not fully compensate the coherency strain. On the other hand, rotations may be finite even in the fully compensated case, and their partitioning does not depend on $d^{B} / d^{A}$, provided $d^{B} \gg z^{m}$. Expressions for the magnitude of the strains and rotations are derived for topologically distinct interfacial defect arrays, (i) dislocations with $\mathbf{b}$ parallel to the substrate terrace, $(\theta=0, \phi=0)$ (ii) dislocations with $\mathbf{b}$ inclined to the substrate terrace, $(\theta=0, \phi \neq 0)$ (iii) disconnections $(\theta \neq 0, \phi \neq 0)$, and (iv) mixed dislocation/disconnection arrays, $(\theta \neq 0, \phi \neq 0)$. Case (iv) is unlike the others in the sense that two characteristic distances arise, associated with the misfit dislocation and disconnection spacings. Non-linear treatments are presented, and shown to correspond with simpler linear versions for small values of $\theta$ and $\phi$. The analysis is applied to one example of each of the four cases, and excellent agreement is obtained between calculated and experimentally observed values.

\section{Appendix A. Reference states}

The reference states are defined by the dichromatic patterns of interfacial symmetry theory [20]. The natural dichromatic pattern is created by the union of the two crystal lattices with terrace planes aligned to be parallel. The Burgers vectors of the interface coherency dislocations are defined with respect to this reference. The 
coherent dichromatic pattern is the union when the lattices are uniaxially strained into coherence on the terraces, and the Burgers vectors and step heights $h$ of disconnections and misfit dislocations are defined in this reference state.

While we consider the static interface here, the defect content of the interface can also be determined from the lattice transformation between the two crystals. The defect content per unit length, $\mathbf{b}^{c}$, of a coherent interface with respect to the natural reference can be calculated from the Frank-Bilby equation as follows

$$
\mathbf{b}^{c}=\left({ }_{c} \mathbf{B}_{n}^{-1}-{ }_{c} \mathbf{A}_{n}^{-1}\right) \mathbf{v}
$$

where $\mathbf{v}$ is a unit length probe vector along the coherent terrace, the matrix $_{c} \mathbf{A}_{n}$ represents the homogeneous deformation that transforms the natural Alattice into the coherent A-lattice, and similarly for ${ }_{c} \mathbf{B}_{n}$.

Appendix B. Coherency strain

The partitioning of coherency strains arises from the condition that the net Newtonian force must vanish at equilibrium on a plane normal to the interface. This corresponds to a balance of stresses. In turn the stresses in general would be given by an expression containing first order and higher order displacement derivatives, with coefficients corresponding to higher order elastic constants. In the small strain limit only the Hooke's law, linear term is significant. The stress is then linear in true strain so that the stress balance gives the strain balance

$$
-\varepsilon_{y y}^{A}=-\ln \left(a^{i} / a^{A}\right)=\varepsilon_{y y}^{B}=\ln \left(a^{i} / a^{B}\right),
$$

which yields for the interface strain

$$
a^{i}=\left(a^{A} a^{B}\right)^{1 / 2} .
$$

Thus $a^{i}$ is the geometric mean of $a^{A}$ and $a^{B}$. Since $<a>=\left(a^{A}+a^{B}\right) / 2$ is the arithmetic mean and $\Delta a=a^{A}-a^{B}$, we have

$$
<a>^{2}=\left(a^{i}\right)^{2}+(\Delta a)^{2} / 4 .
$$




\section{Appendix C. Crystal rotations}

In order to simplify the following equations, which are understood to apply to the $x^{\prime}, y^{\prime}, z^{\prime}$ coordinates of figure 7 , we drop the primes. For a single tilt dislocation $b_{z}$ in the array spaced by $\lambda$, at the origin in the coordinates of figure 7 , the displacement fields as given by equation (3-45) in [16], are 


$$
\begin{aligned}
& u_{y}=\frac{b_{z}}{2 \pi}\left[\tan \frac{z}{y}+\frac{y z}{2(1-v)\left(y^{2}+z^{2}\right)^{2}}\right], \\
& u_{z}=-\frac{b_{z}}{2 \pi}\left[\frac{(1-2 v)}{4(1-v)} \ln \left(y^{2}+z^{2}\right)+\frac{\left(y^{2}+z^{2}\right)}{4(1-v)\left(y^{2}+z^{2}\right)^{2}}\right],
\end{aligned}
$$

where $v$ is Poisson's ratio. This gives the rotation

$$
\omega_{z y}=\frac{1}{2}\left(\frac{\partial u_{z}}{\partial y}-\frac{\partial u_{y}}{\partial z}\right)=\frac{b_{z}}{2 \pi}\left[\frac{-z}{\left(y^{2}+z^{2}\right)}+\frac{z\left(z^{2}-y^{2}\right)}{2(1-v)\left(y^{2}+z^{2}\right)^{2}}\right] .
$$

In reduced coordinates $Z=z / \lambda$ and $Y=y / \lambda$, this expression becomes

$$
\omega_{z y}=\frac{b_{z}}{2 \pi \lambda}\left[\frac{-Z}{\left(Y^{2}+Z^{2}\right)}+\frac{Z\left(Z^{2}-Y^{2}\right)}{2(1-v)\left(Y^{2}+Z^{2}\right)^{2}}\right] .
$$

For the nth dislocation in the array, $Y$ becomes $Y+n$ and one can sum the rotations over the $n$ dislocations from $n=-\infty$ to $+\infty$ using equations (19-72) and (19-73) in [16]. The result is

$$
\omega_{z y}=\frac{b_{z}}{2 \lambda}\left[\frac{-\sinh 2 \pi Z}{(\cosh 2 \pi Z-\cos 2 \pi Y)}+\frac{\pi Z(\cosh 2 \pi Z \cos 2 \pi Y-1)}{2(1-v)(\cosh 2 \pi Z-\cos 2 \pi Y)^{2}}\right] .
$$

The local rotations obviously vary considerably when $|Z|<1$, i.e. when $|z|<\lambda$. For large $Z$ the second term in brackets vanishes and the first term becomes unity. Hence, the rotation $\phi$ properly partitions: $\omega_{z y}=\phi / 2=-b_{z} / 2 \lambda$ for $z>>1$ and $\omega_{z y}=-\phi / 2=b_{z} / 2 \lambda$ for $z<<-1$.

\section{References}

[1] F.C. Frank and J.H. van der Merwe, Proc. Roy. Soc. (London) 198 (1949) p.205.

[2] R. Beanland, C.J. Kiely and R.C. Pond, in Handbook on Semiconductors, T.S.Moss and S.Mahajan eds., North- Holland, Amsterdam, 1994 p.1149.

[3] S.C. Jain, A.H. Harker and R.A. Cowley, Phil. Mag. A75 (1997) p.1461.

[4] E.A. Stach, R. Hull, R.M. Tromp, F.M. Ross, M.C. Reuter and J.C. Bean, Phil. Mag. A80 (2000) p. 2159. 
[5] L.B. Freund and S. Suresh, Thin Film Materials, Cambridge University Press, Cambridge, 2003.

[6] O. Igarishi, J. Appl. Phys.42 (1971) p.4035.

[7] J.Nagai, J. Appl. Phys. 45 (1974) p. 3789.

[8] G.H.Olsen and R.T.Smith, Phys. Stat. Sol. (a) 31 (1975) p.739.

[9] O. Igarishi, Jap. J. Appl. Phys.15 (1976) p.1435.

[10] W.E. Hoke, P.J. Lemonias and R. Traczewski, Appl. Phys.Letts. 44 (1984) p.1046.

[11] J.H. Claassen, S.A. Wolf, S.B. Quadri and L.D. Jones, J.Crystal Growth, 81 (1987) p.557.

[12] M.Shigeta, Y.Fujii, A.Ogara, K. Furukawa, A. Suzuki and S. Nakajima, J.Crystal Growth 93 (1988) p.766.

[13] G. Bai, D.N. Jamieson, M.A. Nicolet and T. Vreeland, in Epitaxy of Semiconductor Layered Structures, R.T. Tung, L.R. Dawsonand, R.L. Gunshor, eds. Materials Research Society Symposium Proceedings 102, Materials Research Society, Pittsburgh, 1988 p.259.

[14] R.Du and C.P. Flynn, J. Cond. Matter 2 (1990) p.1335.

[15] M. Aindow and R.C. Pond, Phil. Mag. 63 (1991) p.667.

[16] J. Dundurs, in Mathematical Theory of Dislocations, T. Mura ed.,Am. Soc. Mech. Engin., New York, 1969, p. 70.

[17]. J.P. Hirth, D.M. Barnett and J. Lothe, Phil. Mag. 40A (1979) p.39.

[18] J.W. Matthews and A.E. Blakeslee, J. Cryst. Growth. 29 (1975) p.273.

[19] R.C. Pond, S. Celotto and J.P. Hirth, Acta Mater.51 (2003) p.5385.

[20] R.C. Pond, X. Ma, Y.W. Chai and J.P. Hirth, in Dislocations in Solids,

F.R.N. Nabarro and J.P. Hirth, eds., Vol. 13, Elsevier, Amsterdam, 2007, p.225.

[21] M.S. Wechsler, D.S. Lieberman and T.A. Read, Trans AIME 197 (1953) p.1503.

[22] J.S. Bowles and J.K. Mackenzie, Acta Metall. 2 (1954) p.129.

[23] R.C. Pond, in Dislocations in Solids. F.R.N. Nabarro, ed., Vol. 8, NorthHolland, Amterdam, 1989, p.1.

[24]. J.P. Hirth and R.C. Pond, Acta Mater. 44 (1996) p.4749.

[25] J.W. Matthews, Phil. Mag. 13 (1966) p.1207.

[26] E.R. Thompson and K.R. Lawless, Appl. Phys. Lett. 9 (1966) p.138.

[27] J.W. Matthews and J.L. Crawford, Thin Solid Films 5 ((1970) p.187. 
[28] K. Shinohara and J.P. Hirth, Thin Solid Films 16 (1973) p.346.

[29] A.M. Beers and E.J. Mittemeijer, Thin Solid Films 48 (1978) p.367.

[30] R. Hull and J.C. Bean, Appl. Phys. Lett. 54 (1989) p.925.

[31] Y.R. Xing, R.W. Devenish, T.B. Joyce, C.J. Kiely, T. Bullough and P.J. Goodhew, Appl. Phys. Letts. 60 (1992) p.616.

[32] J.W. Matthews, Phil Mag. 29 (1974) p.797.

[33] J.P. Hirth and X. Feng, J. Appl. Phys. 67 (1990) p.3343.

[34] J.P. Hirth and J. Lothe, Theory of Dislocations, Krieger, Melbourne, FL, 1992, Chap. 19.

[35]. K. Shinohara and J. P. Hirth, Phil. Mag. 27 (1973) p.883.

[36] R.C. Pond and J.P. Hirth, Phil. Mag. in press.

[37] J.P. Hirth, R.C. Pond and J. Lothe, Acta Mater. 54 (2006) p.4237.

[38] G.A. Bassett, Phil. Mag. 3 (1958) p.72.

[39] B.K. Chakraverty and G.M. Pound, Acta Metall. 12 (1964) p.851.

[40] J. Hornstra and W.J. Bartels, J. Cryst. Growth 44 (1978) p. 513.

[41] D. Eaglesham, M. Aindow and R.C. Pond, Materials Research Society Symposium Proceedings, 116, Materials Research Society, Pittsburgh, 1987) p.267.

[42] P.J. Dugdale, R.C. Pond and R. Beanland, Mat. Sci. Forum 126 (1993) p.281.

[43] K. Han, J.D. Embury, J.J. Petrovic and G.C. Weatherly, Acta Mater. 46 (1998) p.4691.

[44] D. Klenov, Ph.D. Thesis, University of Birmingham, U.K., 2002.

[45] A.J. Schwartz, H. Cynn, K.J.M. Blobaum, M.A. Wall, K.T. Moore, W.J. Evans, D.L. Farber, J.R. Jeffries and T.B. Massalski, Prog. in Mat. Sci. 54 (2009) p.909.

[46] J.M. Howe, R.C. Pond and J.P. Hirth, Prog. in Mat. Sci. 54 (2009) p.792.

[47] C. Hammond and P.M. Kelly, Acta Metall. 17 (1969) p.869.

[48] M. Aindow, D. Eaglesham and R.C. Pond, Inst. Phys. Conf. Ser. 93, vol. 2 (1988) p. 405.

[49] D. Dimos, P. Chaudhari, J. Mannhart and F.K. Le Goues, Phys. Rev. Lett. 61 (1988) p.219.

[50] D. Mitlin, A. Misra, T.E. Mitchell, J.P. Hirth and R.G. Hoagland, Phil. Mag. 85 (2005) p. 3379. 